\title{
Earth's Gravity Gradient and Eddy Currents Effects on the Rotational Dynamics of Space Debris Objects: Envisat Case Study
}

\author{
Natalia Ortiz Gómez \\ Southampton University, Highfield SO17 1BJ United Kingdom \\ Dr. Scott J.I. Walker \\ Southampton University, Highfield SO17 1BJ United Kingdom
}

\begin{abstract}
The Space Debris population has grown rapidly over the last few decades with the consequent growth of impact risk between current objects in orbit. Active Debris Removal (ADR) has been recommended to be put into practice by several National Agencies in order to remove objects that pose the biggest risk for the space community. The most immediate target that is being considered for ADR by the European Space Agency is the Earth-observing satellite Envisat. In order to safely remove such a massive object from its orbit, a capturing process followed by a controlled reentry is necessary. However, current ADR methods that require physical contact with the target have limitations on the maximum angular momentum that can be absorbed and a de-tumbling phase prior to the capturing process may be required. Therefore, it is of utmost importance for the ADR mission design to be able to predict accurately how the target will be rotating at the time of capture. This article analyses two perturbations that affect an object in Low Earth Orbit (LEO), the Earth's gravity gradient and the eddy currents induced by the Earth's magnetic field. The gravity gradient is analysed using the equation of conservation of total energy and a graphical method is presented to understand the expected behaviour of any object under the effect of this perturbation. The eddy currents are also analysed by studying the total energy of the system. The induced torque and the characteristic time of decay are presented as a function of the object's magnetic tensor. In addition, simulations were carried out for the Envisat spacecraft including the gravity gradient perturbation as well as the eddy currents effect using the International Geomagnetic Reference Field IGRF-11 to model the Earth's magnetic field. These simulations show that the combined effect of these two perturbations is a plausible explanation for the rotational speed decay observed between April 2013 and September 2013.
\end{abstract}

Keywords: Space Debris; Tumbling; Gravity Gradient; Eddy Currents; Envisat

\section{Introduction}

Several studies carried out at National Agencies and Universities (Kessler et al., 2010) foresee an increase of the space debris population in the upcoming decades. This has led to the development of new guidelines for the design of space missions in order to limit the generation of new orbital debris (ESA, 2005; NASA, 2011). Some of the rules dictated by ESA and NASA include a systematic passivation of every stage left in orbit in order to minimize break-ups and a maximum post-mission lifetime of 25 years. In addition, National Agencies recommend to combine both post mission disposal measures and Active Debris Removal (ADR) in order to stop the population growth (UNOOSA, 2012).

Current ADR methods can be classified into contact and contactless methods (Wormnes et al., 2013). At present,

Email addresses: N.Ortiz-Gomez@soton.ac.uk (Natalia Ortiz Gómez), sjiw@soton.ac.uk (Dr. Scott J.I. Walker) one of the main challenges of the contact ADR methods is to be able to capture a non-cooperative rotating object. Rigid capture systems, such as the robotic arm, are not able to grab and stabilize objects which are rotating faster than a few degrees per second (Nishida et al., 2011). Other capturing systems such as the harpoon or the net may have difficulties in capturing fast rotating objects due to the possible entanglement of the tether with the target. Some of these systems require a zero relative rotational velocity state between the chaser and the target before the docking/berthing phase. This step may pose difficulties if the target has a complex rotational motion, such as a rotation about the three principal axes. For these reasons, it is crucial to predict accurately what will be the rotational state of the space debris object at the time of capture in order to choose the most suitable ADR method for the mission.

Optical and radar observations carried out to determine the rotational state of these objects are on the in- 
crease. These initiatives will help understand what are the expected space debris rotational states depending on their orbit, shape configuration and materials. Up-to-date observed rotational speeds vary greatly, from less than $1 \mathrm{deg} / \mathrm{sec}$ up to $60 \mathrm{deg} / \mathrm{sec}$ or even more (Papushev et al., 2009). However, experts still have difficulty in finding a physical explanation for some of the observed motions of these objects. For instance, optical and radar measurements carried out in 2012 for the Envisat spacecraft showed intrinsic rotations of 0.2 degrees/sec along the axis normal to the orbital plane which coincided approximately with the minimum axis of inertia of the spacecraft (Lemmens et al., 2013). However, later radar measurements showed a fast increase of the rotational speed at the beginning of 2013, up to $2.8 \mathrm{deg} / \mathrm{sec}$. In April 2013, the rotational speed began to decrease again at a lower pace (with a rate of period increase equal to $36.7 \mathrm{~ms} /$ day) until the last observation made in September 2013 (Kucharski et al., 2014). In addition, a change in the attitude was observed which now has its minimum axis of inertia approximately pointing in the along track direction of the orbit. This behavior is yet to be explained. Its understanding is of the utmost importance in order to predict accurately its future attitude motion for the ADR mission design, planned for 2021 by ESA (Biesbroek et al., 2013).

The present article focuses on two of the most important perturbations that affect the rotational dynamics of space debris objects in LEO: the Earth's gravity gradient and the eddy currents induced by the Earth's magnetic field (Ojakangas et al., 2012; Praly et al., 2012). The torques induced by these perturbations will depend on the physical properties of the spacecraft. The gravity gradient torque will affect bodies with a non-isotropic inertia tensor and it is proportional to the inverse cubed of the distance to the Earth (Hughes, 2004). In addition, the Earth's magnetic field will induce eddy current loops on rotating conductive bodies and their influence also decays with the cube of the distance to the Earth (Hughes, 2004; Smith, 1965). The eddy current torques will be more noticeable in those objects that have more conductive materials electrically connected. Observations carried out for rotating upper stages show that the eddy current torques can outweigh the atmospheric drag perturbations at heights lower than $500 \mathrm{~km}$ (Williams et al., 1978). Additional environmental perturbations such as the solar radiation pressure usually provides the dominating contribution at higher altitudes (synchronous altitude). A comparison between these perturbations can be found in (Ojakangas et al., 2012; Gerlach, 1965).

In this article, the Earth gravity gradient and the eddy currents are analysed in detail given their importance in LEO orbits. A case study is carried out for the Envisat spacecraft and the results are compared with the observations carried out in 2013 (Kucharski et al., 2014) .

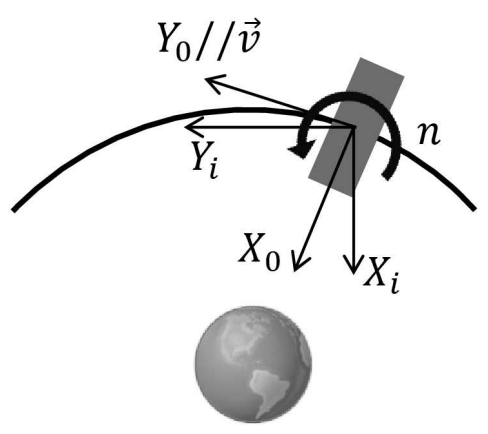

Figure 1: Rotation between the orbital and inertial reference frames.

\section{Reference frames}

Three different reference frames are used throughout the document:

- Inertial Reference Frame: This reference frame has its origin in the centre of the Earth and its axis coincide with the J2000 Inertial Reference Frame. The nomenclature for this reference system is $\left(X_{i}, Y_{i}, Z_{i}\right)$.

- Orbital Reference Frame: This reference frame has its origin in the centre of gravity (COG) of the body in orbit. The $Y_{0}$ axis points in the along track direction, parallel to the velocity of the body $\left(\vec{j}_{0}=\right.$ $\left.\frac{\vec{v}}{|\vec{v}|}\right)$ and the $Z_{0}$ axis is perpendicular to the orbital plane, parallel to the orbital angular momentum. Finally, the $X_{0}$ axis completes the reference frame $\left(\vec{i}_{0}=\vec{j}_{0} \times \vec{k}_{0}\right)$. In the case of a circular orbit, the $X_{0}$ axis coincides with the nadir direction.

- Body Frame: This reference frame has its origin in the COG of the body and coincides with the principal axes of inertia of the object. The nomenclature for this reference system is $\left(X_{b}, Y_{b}, Z_{b}\right)$.

In this article, only circular orbits are considered. For this type of orbit, the relationship between the orbital and the inertial reference frame is a rotation around the $-Z_{0}$ axis (axis perpendicular to the orbital plane) at a pace equal to the angular velocity $n=\frac{2 \pi}{T}$, where $T$ is the orbital period. Both frames are represented in figure 1.

In order to rotate from the orbital reference frame to the body frame, a 1-3-2 Euler transformation is carried out which consists of a sequence of three rotations $(\psi, \theta, \phi)=$ (Yaw,Pitch,Roll). Figure 2 shows a simplified representation of the Euler angles assuming that they are small.

The rotation matrix to lead from the body frame to the orbital reference frame is shown in (1).

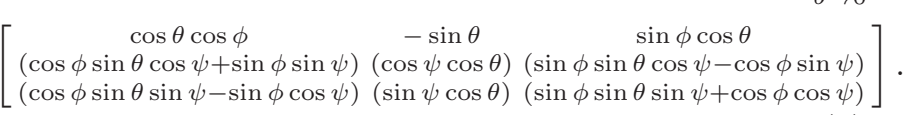




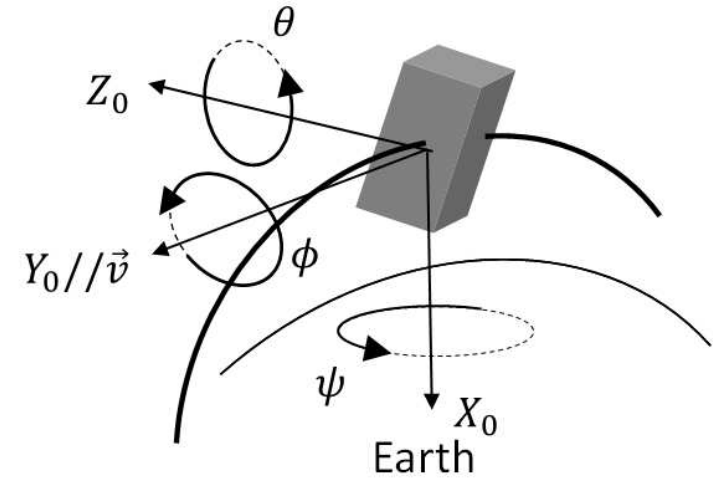

Figure 2: Orbital Reference Frame and Euler Angles

The angular velocity vector between the body reference frame and the orbital reference frame, expressed in the body frame is shown in (2).

$$
\begin{array}{r}
\omega_{b 0}=(\dot{\psi} \cos \theta \cos \phi-\dot{\theta} \sin \phi) \overrightarrow{i_{b}}+ \\
(\dot{\phi}-\dot{\psi} \sin \theta) \overrightarrow{j_{b}}+(\dot{\psi} \sin \phi \cos \theta+\dot{\theta} \cos \phi) \overrightarrow{k_{b}}
\end{array}
$$

The rotational velocity between the orbital reference frame and inertial reference frame is shown in (3).

$$
\overrightarrow{\omega_{0 i}}=\overrightarrow{\omega_{n}}=-n \vec{k}_{0}=-\frac{2 \pi}{T} \overrightarrow{k_{0}}=-\sqrt{\frac{\mu}{R^{3}}} \overrightarrow{k_{0}},
$$

where $T$ is the orbital period and $R$ is the distance from the center of the Earth to the centre of gravity of the body in orbit.

Therefore, the angular velocity of the body reference frame with respect to the the inertial reference frame, expressed in the body frame is shown in (4).

$$
\omega_{b \rightarrow i}=
$$

$(\dot{\psi} \cos \theta \cos \phi-\dot{\theta} \sin \phi-n(\cos \phi \sin \theta \sin \psi-\sin \phi \cos \psi)) \overrightarrow{i_{b}}+$

$$
(\dot{\phi}-\dot{\psi} \sin \theta-n(\sin \psi \cos \theta)) \overrightarrow{j_{b}}+
$$

$(\dot{\psi} \sin \phi \cos \theta+\dot{\theta} \cos \phi-n(\sin \phi \sin \theta \sin \psi+\cos \phi \cos \psi)) \overrightarrow{k_{b}}$.

It should be noted that the Euler angles are advised to be used in the integration process of the Euler equations due to the singularity problems it entails (Hughes, 2004). This can be observed easily in the rotation matrix between the body frame and the orbital reference frame (1) which is singular for certain values of the Euler angles. However, this local representation will be employed for the theoretical analysis of the gravity gradient and its later graphical representation, as it is very helpful for the physical understanding of the equations and the interpretation of the results. For the integration process, a more appropiate representation was employed based on quaternions.

\section{Gravity gradient}

The attitude dynamics of a rigid body subject to the Earth's gravity gradient is a well-studied problem. Here, a graphical interpretation of the attitude evolution of real objects subject to the Earth's gravity gradient is presented. This will help to understand the behaviour of the system when additional dissipative effects are included, such as the eddy currents considered later. Existing studies on the Earth's gravity gradient usually focus on a purely mathematical analysis of the stability conditions of the system's equilibrium points (Gerlach, 1965; Siahpush et. al., 1987; Hughes, 2004). The presented graphical display of the results allows the reader to easily observe how the system evolves, oscillating around equilibrium points or abandoning the region of marginal stability (see section 3.4.1). Additionally, it enables the reader to easily understand under which kinetic energy conditions, the system has a bounded or unbounded motion. In section 3.4.1 an analysis is presented for the Envisat spacecraft and in section 3.4.2 an additional example is included were the equilibrium states are not points but linear varieties.

\subsection{General equations}

The Earth's gravitational force is inversely proportional to the square of the distance to the centre of gravity of the Earth. Therefore, if an object in orbit is divided into small increments of mass $d m$, each one of them will be subjected to a differential force $d F$ as shown in (5).

$$
d \vec{F}=-\mu \frac{\vec{\rho} d m}{|\vec{\rho}|^{3}}, \quad \vec{\rho}=\vec{R}+\vec{r},
$$

where $\mu$ is the Earth's gravitational constant (i.e., $0.3986 \times$ $\left.10^{15} \mathrm{~m}^{3} / \mathrm{s}^{2}\right), \vec{\rho}$ is the distance from the Earth's centre of mass to the differential mass $d m, \vec{R}$ is the distance from the Earth's centre of mass to the centre of mass of the body in orbit and $\vec{r}$ is the distance from the centre of mass of the body to the differential mass.

The components of the gravity gradient's torque in the body frame, as a function of the Euler angles sequence defined in section 2 are presented in (6), (7) and (8),

$$
\begin{aligned}
T_{x} & =\frac{3 \mu}{2 R^{3}}\left(I_{y y}-I_{z z}\right) \sin 2 \theta \sin \phi \\
T_{y} & =\frac{3 \mu}{2 R^{3}}\left(I_{x x}-I_{z z}\right) \sin 2 \phi \cos ^{2} \theta, \\
T_{z} & =\frac{3 \mu}{2 R^{3}}\left(I_{x x}-I_{y y}\right) \sin 2 \theta \cos \phi .
\end{aligned}
$$

The mathematical process followed to obtain the gravity gradient torque can be easily found in the literature ((Gerlach, 1965),(Hughes, 2004)). Using the Euler equations (Hughes, 2004), the differential equations obtained are presented in (9), (10) and (11),

$$
\begin{aligned}
I_{x x} \dot{\omega}_{x}+\left(I_{z z}-I_{y y}\right) \omega_{y} \omega_{z} & =-\frac{3 \mu}{2 R^{3}}\left(I_{z z}-I_{y y}\right) \sin 2 \theta \sin \phi \\
I_{y y} \dot{\omega}_{y}+\left(I_{x x}-I_{z z}\right) \omega_{x} \omega_{z} & =-\frac{3 \mu}{2 R^{3}}\left(I_{z z}-I_{x x}\right) \sin 2 \phi \cos ^{2} \theta
\end{aligned}
$$


$I_{z z} \dot{\omega}_{z}+\left(I_{y y}-I_{x x}\right) \omega_{x} \omega_{y}=-\frac{3 \mu}{2 R^{3}}\left(I_{y y}-I_{x x}\right) \sin 2 \theta \cos \phi$

In the previous differential equations $(9,10,11)$, the variables $\omega_{x}, \omega_{y}$ and $\omega_{z}$ are the components of the rotational velocity vector between the body frame and the inertial reference frame expressed in the body frame (expression 4). In addition, the parameters $I_{x x}, I_{y y}, I_{z z}$ are the components of the inertia tensor in the principal axes of inertia of the body.

\subsection{Energy conservation}

The gravity gradient is a conservative force and therefore, the total energy of the system must remain constant. This conservation law will help analyse the motion of the space debris object. The kinetic energy expressed with the components of the rotational velocity vector in the body frame is shown in (12),

$$
K=\frac{1}{2}\left(I_{x x} \omega_{x}^{2}+I_{y y} \omega_{y}^{2}+I_{z z} \omega_{z}^{2}\right) .
$$

Using Euler equations, the derivative of the kinetic energy is obtained. Since $(\vec{\omega} \times I \vec{\omega}) \cdot \vec{\omega}=0$ and $\dot{I}=0$, it holds that:

$$
\dot{K}=\vec{T} \cdot \vec{\omega}=I \dot{\vec{\omega}} \cdot \vec{\omega}+(\vec{\omega} \times I \vec{\omega}) \cdot \vec{\omega}=I \vec{\omega} \cdot \dot{\vec{\omega}}
$$

where $I$ is the inertia tensor of the body expressed in the body frame. The potential energy of the gravity force is defined in (14).

$$
U_{G}=\int-\frac{\mu d m}{|\vec{r}+\vec{R}|} .
$$

Taking into account that $|\vec{r}|<<|\vec{R}|, U_{G}$ can be expanded in a Taylor series and the following expression for the potential energy is reached (Hughes, 2004), including up to second order perturbation terms:

$$
U_{G}=-\frac{\mu m}{R}+\frac{3 \mu}{2 R^{3}} I_{x 0 x 0},
$$

where $I_{x 0 x 0}$ is the moment of inertia with respect to the orbital axis $\vec{i}_{0}$ (see appendix A). The first term that affects the rotational dynamics of a body subject to the Earth's gravity gradient is shown in (16).

$$
\begin{array}{r}
U_{G G}=\frac{3 \mu}{2 R^{3}} I_{x 0 x 0}= \\
\frac{3 \mu}{2 R^{3}}\left(I_{x x} \cos ^{2} \theta \cos ^{2} \phi+I_{y y} \sin ^{2} \theta+I_{z z} \cos ^{2} \theta \sin ^{2} \phi\right) .
\end{array}
$$

It should be noted that $U_{G G}$ only depends on two of the Euler angles, which will later enable 3D figures to be plotted, allowing the stability of the overall system to be analysed.
The next step is the evaluation of the time derivative of $U_{G G}$ to obtain its relationship with that of the kinetic energy $K$.

$$
\begin{array}{r}
\frac{d U_{G G}}{d t}=-\frac{3 \mu}{2 R^{3}}\left[I_{x x}\left(\dot{\phi} \sin 2 \phi \cos ^{2} \theta+\dot{\theta} \sin 2 \theta \cos ^{2} \phi\right)\right. \\
\left.-I_{y y} \dot{\theta} \sin 2 \theta+I_{z z}\left(\dot{\theta} \sin 2 \theta \sin ^{2} \phi-\dot{\phi} \sin 2 \phi \cos ^{2} \theta\right)\right]= \\
-\vec{T} \cdot \vec{\omega}_{b 0} .
\end{array}
$$

The scalar product $\vec{T} \cdot \vec{\omega}$ is evaluated as shown in (18).

$$
\begin{array}{r}
\vec{T} \cdot \vec{\omega}=\vec{T} \cdot \vec{\omega}_{b 0}+\vec{T} \cdot \overrightarrow{\omega_{n}}= \\
-\frac{d U_{G G}}{d t}+\vec{T} \cdot \overrightarrow{\omega_{n}} .
\end{array}
$$

The term $\overrightarrow{\omega_{n}}$ is the rotational velocity between the orbital reference frame and the inertial reference frame. The product $\vec{T} \cdot \overrightarrow{\omega_{n}}$ acquires the form presented in (19),

$$
\begin{array}{r}
\vec{T} \cdot \overrightarrow{\omega_{n}}= \\
n \frac{3 \mu}{2 R^{3}}\left[I_{x x}\left(-\sin \psi \cos \theta \sin 2 \theta-\cos ^{2} \phi \sin 2 \theta \cos \psi\right)\right. \\
+I_{y y}(\cos \psi \sin 2 \theta) \\
\left.+I_{z z}\left(\sin \psi \cos \theta \sin 2 \phi-\sin ^{2} \phi \cos \psi \sin 2 \theta\right)\right]= \\
-n \frac{3 \mu}{R^{3}} I_{x 0 y 0},
\end{array}
$$

where $I_{x 0 y 0}$ is one of the products of inertia of the inertia tensor in the orbital reference frame (see appendix A).

Finally, the time derivative of the energy adopts the form presented in (20),

$$
\frac{d\left(K+U_{G G}\right)}{d t}=\vec{T} \cdot \overrightarrow{\omega_{n}}=-n \frac{3 \mu}{R^{3}} I_{x 0 y 0} .
$$

The term $\left(\vec{T} \cdot \overrightarrow{\omega_{n}}\right)$ can also be expressed as a derivative of a potential function. This term appears due to the fact that a non-inertial reference frame is used. Landau \& Lifshitz Vol 01 Mechanics Book (Landau, 1976) provide the expression that relates the energies in an inertial reference frame and a non-inertial reference frame (21),

$$
E=E_{\text {inertial }}+\Delta E=E_{\text {inertial }}-\vec{H} \vec{\omega}_{0 i}
$$

In the case at hand $\omega_{0 i}=n \overrightarrow{k_{0}}$ is the angular velocity vector between the orbital reference frame and body frame. The vector $\vec{H}$ is the angular momentum of the system:

$$
\vec{H}=I \overrightarrow{\omega_{b i}}=I_{x x} \omega_{x} \overrightarrow{i_{b}}+I_{y y} \omega_{y} \overrightarrow{j_{b}}+I_{z z} \omega_{z} \overrightarrow{k_{b}} .
$$

Therefore, the term $\Delta E$ can be expressed as shown in (23). 


$$
\Delta E=-\left(I \vec{\omega}_{b i}\right) \cdot \vec{\omega}_{n}=n\left(I \vec{\omega}_{b i}\right) \cdot \overrightarrow{k_{0}} .
$$

In appendix $\mathrm{B}$, the mathematical derivation that proves $-\frac{d \Delta E}{d t}=\vec{T} \cdot \overrightarrow{\omega_{n}}$ is included.

Finally, the energy conservation for the rotational dynamics of a body due to the gravity gradient effect is expressed as shown in (24),

$$
E=K+U_{G G}+\Delta E=\text { Constant },
$$

where:

$$
K=\frac{1}{2}\left(I_{x x} \omega_{x}^{2}+I_{y y} \omega_{y}^{2}+I_{z z} \omega_{z}^{2}\right)
$$

$$
\begin{array}{r}
U_{G G}=\frac{3}{2} n^{2} I_{x 0 x 0}= \\
\frac{3}{2} n^{2}\left(I_{x x} \cos ^{2} \theta \cos ^{2} \phi+I_{y y} \sin ^{2} \theta+I_{z z} \cos ^{2} \theta \sin ^{2} \phi\right)
\end{array}
$$

$$
\Delta E=n\left(I \vec{\omega}_{b i}\right) \cdot \overrightarrow{k_{0}}=-\left(I \vec{\omega}_{b i}\right) \cdot \vec{\omega}_{n}
$$

The equation of energy conservation can be regrouped into two terms using a pseudo-kinetic energy $K^{\prime}$. The function $K^{\prime}$ gathers all the terms that are function of the rotational velocity $\vec{\omega}_{b i}$. The potential energy $U_{G G}$ takes into account terms which only depend on the position parameters $(\vec{\Theta}=[\psi, \theta, \phi])$. For the sake of simplicity, $\vec{\omega}_{b i}$ will be expressed hereinafter as $\vec{\omega}$.

$$
\begin{array}{r}
E=U_{G G}(\vec{\Theta})+K^{\prime}(\vec{\omega}), \\
K^{\prime}=K+\Delta E=\frac{1}{2} \vec{\omega}^{t} I \vec{\omega}+n \vec{k}_{0}^{t} I \vec{\omega}
\end{array}
$$

It is observed that the pseudo-kinetic energy $K^{\prime}$ can be both positive and negative. Therefore, the constant value of the energy $E$ does not guarantee the movement of the system to those regions where $U_{G G}(\vec{\Theta})<E$. However, an offset can be added to the pseudo-kinetic energy equal to its minimum value in each state, $K_{\text {min }}^{\prime}(\vec{\Theta})$, to guarantee that it will be non-negative. The minimum value of the function is given by (29).

$$
\begin{array}{r}
\frac{\partial K^{\prime}}{\partial \dot{\vec{\omega}}}=\overrightarrow{0} \rightarrow \\
I \vec{\omega}+n I \vec{k}_{0}=\overrightarrow{0} \rightarrow \vec{\omega}=-n \vec{k}_{0}=\vec{\omega}_{n} .
\end{array}
$$

Note that this minimum of $K^{\prime}$ occurs when $\vec{\omega}_{b 0}=\overrightarrow{0}$ and hence, for the stationary states $(\dot{\vec{\Theta}}=[\dot{\psi}, \dot{\theta}, \dot{\phi}])=\overrightarrow{0}$. Therefore, $K^{\prime}-K_{\text {min }}^{\prime}$ is strictly positive except for $\dot{\vec{\Theta}}=\overrightarrow{0}$, when it is zero. The offset $K_{\text {min }}^{\prime}$ is shown in (30),

$$
K_{\text {min }}^{\prime}(\vec{\Theta})=\frac{n^{2}}{2} \vec{k}_{0}^{t} I \vec{k}_{0}-n^{2} \vec{k}_{0}^{t} I \vec{k}_{0}=-\frac{n^{2}}{2} I_{z 0 z 0}
$$

The energy equation remains unchanged if the same magnitude is added and subtracted as follows:

$$
E=\left(K^{\prime}+\left|K_{\min }^{\prime}\right|\right)+\left(U_{G G}-\left|K_{\min }^{\prime}\right|\right)
$$

Now, the new kinetic energy $K^{\prime}+\left|K_{\text {min }}^{\prime}\right|$ will always be greater than or equal to 0 . It becomes zero for $\dot{\vec{\Theta}}=\overrightarrow{0}$. This result will enable intuitive reasoning of the system's motion based on thresholds of the potential energy considered. Therefore the energy equation is grouped into two terms: the new kinetic energy will be refered to as noninertial kinetic energy, $K_{N I}$, and the potential energy will be refered to as non-inertial potential energy, $U_{N I}$. Equations (32), (33) and (34) show the final expressions for the total energy of the system $E$, the non-inertial kinetic energy $K_{N I}$ and the non-inertial potential energy $U_{N I}$ respectively,

$$
\begin{gathered}
E=K_{N I}+U_{N I}=\text { constant }, \\
K_{N I}=\frac{1}{2} \omega_{b 0}^{t} I \omega_{b 0}, \\
U_{N I}=\frac{1}{2} n^{2}\left(3 I_{x 0 x 0}-I_{z 0 z 0}\right) .
\end{gathered}
$$

Equation (33) is obtained by substituting $\vec{\omega}=\vec{\omega}_{b 0}+\vec{\omega}_{n}$ in $K_{N I}=K^{\prime}+\left|K_{m i n}^{\prime}\right|$. Note that the final expression for (33) corresponds to the kinetic energy in the orbital reference frame which justifies its denomination as "noninertial kinetic energy".

\subsection{Stability analysis}

In order to define the stability around an equilibrium point $X$, a generic ordinary differential equation (ODE) $\dot{x}=f(t, x)$ is considered, where $x$ is a n-dimensional variable. The system is said to be asymptotically stable around a given equilibrium point $X$ in the sense of Lyapunov if:

1. Given any $\epsilon>0$ for any $t_{0} \geq 0$, there exists a $\delta_{1}=\delta_{1}\left(\epsilon, t_{0}\right)$ such that

$$
\left|x\left(t_{0}\right)-X\right|<\delta_{1} \Rightarrow|x(t)-X|<\epsilon, \forall t \geq t_{0} \geq 0,
$$

2. There exists $\delta_{2}\left(t_{0}\right) \geq 0$ for any $t_{0} \geq 0$ such that

$$
\left|x\left(t_{0}\right)-X\right|<\delta_{2} \Rightarrow|x(t)-X| \rightarrow 0 \text { as } t \rightarrow \infty .
$$

The first condition requires that the state trajectory of the system is confined to an arbitrarily small domain (radius $\epsilon$ ) centered at the equilibrium point given an arbitrary initial state which is sufficiently close to the equilibrium point. If a system only complies with the first requirement, it is said to be 'marginally stable'.

The conservative behaviour of the gravity gradient problem given by equation (32), enables a region of marginal stability to be defined where the system evolution may be bounded, depending on the energy potential threshold. This region of marginal stability is defined as a domain 
around an equilibrium point where the function $U_{N I}$ is monotonically increasing. The potential threshold for a given relative or absolute minimum of the non-inertial potential energy $\left(U_{N I}\left(\vec{\Theta}_{U_{N I} m i n}\right)\right)$ is presented in $(37)$,

$$
\begin{array}{r}
\Delta U_{N I}=\min \left(U_{N I}(\vec{\Theta})-U_{N I}\left(\vec{\Theta}_{U_{N I} m i n}\right)\right) \\
\vec{\Theta} \in \text { Region of marginal stability }
\end{array}
$$

where $\vec{\Theta}_{U_{N I} \text { min }}$ is the state vector for which $U_{N I}$ reaches the relative or absolute minimum.

This gives rise to two different possible behaviours of the system under the gravity gradient perturbation. The first one is a "bounded motion". Given that the noninertial kinetic energy $K_{N I}$ is a definitive positive function, this situation will take place when the system initially does not have enough kinetic energy to abandon the region of marginal stability.

The second type of behaviour is an "unbounded motion". A sufficient condition to allow the system to abandon the region of marginal stability is that the non-inertial kinetic energy of the system is above the threshold $\left(K_{N I}>\right.$ $\left.\Delta U_{N I}\right)$. As the $U_{N I}$ function has an absolute maximum equal to $\frac{1}{2} n^{2}\left(3 I_{\max }-I_{\min }\right)$, even for the absolute minimum, its region of marginal stability is finite. Therefore, it can be inferred that the aforementioned "unbounded motion" exists.

However, the system can also have more complex motions if the equilibrium states are not isolated points but linear varieties (see section 3.4.2). In this case, when the system does not have enough kinetic energy, its motion is obliged to stay in the vicinity of the linear varieties. This defines a sub-domain of states which can be reached by the system, more complex than in the case of an isolated equilibrium point. For instance, in the case study (3.4.2), this sub-domain is multiply connected.

The non-inertial potential energy $U_{N I}$, given by (34), has an absolute minimum, which therefore corresponds to the most stable state. Equation (34) shows that the minimum is reached when the inertia about the nadir direction is minimum $I_{x 0 x 0}=I_{\min }$ and the inertia about angular momentum vector of the orbit is maximum $I_{z 0 z 0}=I_{\max }$. This coincides with the well-known equilibrium point given by the gravity gradient (Langford et al., 1964; Hughes, 2004).

The existent gravity gradient stability analyses are usually carried out in the vicinity of the equilibrium points. This gives local information on whether a given orientation of the principal axes of the body is stable or unstable. However, a simple graphical representation of the potential energy values integrated throughout time can be a very powerful tool to observe the overall behaviour of the system and the expected behaviour for any given initial condition (initial attitude and rotational velocity of the space debris object) which need not be close to an equilibrium point.
Two possible graphical representations are suggested to visualise the evolution of the system subject to the gravity gradient.

The first one consists of plotting the 2D plane function $U_{N I}=f\left(i_{x 0 x 0}, i_{z 0 z 0}\right)$, where $i_{x 0 x 0}=I_{x 0 x 0} / I_{\max }$ and $i_{z 0 z 0}=I_{z 0 z 0} / I_{\max }$ are the normalised inertias of the orbital axis $X_{0}$ and $Z_{0}$. The parameters $i_{x 0 x 0}$ and $i_{z 0 z 0}$ will always be positive and will vary between $i_{\text {min }}$ and 1 (minimum and maximum inertias of the body). Figure 3 shows all the boundaries of the domain of definition of this function. The first boundary is given by the minimum and maximum values of the inertias of the rigid body. This defines the rectangle $A C F H$ in figure 3 . The second boundary comes from the fact that the trace of the inertia tensor is constant. This trace, $\operatorname{tr}(I)$, is calculated as:

$$
\operatorname{tr}(I)=I_{x 0 x 0}+I_{y 0 y 0}+I_{z 0 z 0}=I_{\text {max }}+I_{\text {med }}+I_{\text {min }},
$$

where $I_{\text {max }}, I_{\text {med }}, I_{\text {min }}$ are the maximum, intermediate and minimum values of the principal inertias. Now, this equation can be normalised with $I_{\max }$ as shown in (39),

$$
i_{x 0 x 0}+i_{y 0 y 0}+i_{z 0 z 0}=1+i_{m e d}+i_{m i n}
$$

where $i_{\max }=1, i_{\text {med }}=\frac{I_{\operatorname{med}}}{I_{\max }}$ and $i_{\min }=\frac{I_{\min }}{I_{\max }}$. This condition generates two parallel linear boundaries which are at 45 degrees and with negative slope (line $I G$ and $B D)$. The system must remain within these two lines. The two boundaries in the plane $i_{x 0 x 0}, i_{z 0 z 0}$ are the following:

$$
\begin{gathered}
i_{x 0 x 0}+i_{z 0 z 0}=1+i_{m e d}+i_{m i n}-i_{y 0 y 0} \rightarrow \\
\rightarrow\left\{\begin{array}{l}
i_{x 0 x 0}+i_{z 0 z 0} \leq 1+i_{m e d} \text { Boundary BD } \\
i_{x 0 x 0}+i_{z 0 z 0} \geq i_{m e d}+i_{m i n} \text { Boundary IG }
\end{array}\right.
\end{gathered}
$$

The third boundary is given by the energy equation (32) and it corresponds to the line $G E$ in figure 3. Taking into account that $K_{N I} \geq 0$, the inequality (42) is reached,

$$
\begin{gathered}
E-U_{N I} \geq 0, \\
i_{z 0 z 0}-3 i_{x 0 x 0}+\frac{E}{\frac{n^{2}}{2} I_{\max }} \geq 0 .
\end{gathered}
$$

It must be noted that the energy and trace boundaries can be tangent or outside the rectangle $A C F H$ and therefore, the restraint may not be active. Depending on the specific data of each problem, this generic domain will follow different patterns. In addition, depending on the initial conditions, only a part of the allowed domain will be filled by the state of the system. Several examples are presented later in the article.

The second possible graphical representation consists in plotting the function $U_{G G}=f(\theta, \phi)$. This term of the gravity potential only depends on two Euler angles which enables $2 \mathrm{D}$ representations to be used. It should be highlighted that this representation only takes into account 


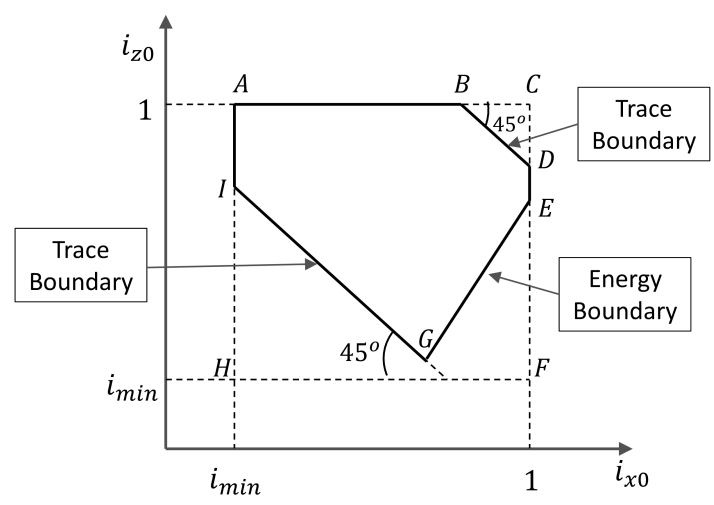

Figure 3: Boundaries of the domain of definition of the function $U_{N I}=f\left(i_{x 0 x 0}, i_{z 0 z 0}\right)$

part of the $U_{N I}$ function. Nevertheless, the following examples (section 4.4) show that this graphical representation can be very useful when dissipative effects such as the eddy currents are involved in order to visualise the possible asymptotic convergence that may arise.

Due to the multivaluated behaviour of the Euler angles, it is convenient to carry out this graphical representation, restricting the angles to a specific interval. In the following simulations, the Euler angles were restricted to the interval $[-180,+180]$ degrees by a congruence of modulus of 360 degrees. This also gives valuable information concerning the evolution of the system and shows the 'unbounded' and 'bounded' behaviours. The following simulations show these methodologies in more detail.

\subsection{Simulations}

Two case studies are analysed with different inertia matrices. The first one corresponds to the Envisat spacecraft and the second one corresponds to a spin stabilised inertialike spacecraft. This second example was included in the article in order to show a case of equilibrium states in the form of linear varieties, which differ greatly from that of the Envisat spacecraft.

\subsubsection{Case study: Envisat}

The European Space Agency is planning a mission to capture and de-orbit the Envisat spacecraft in 2021 (Biesbroek et al., 2013). In order to choose the most suitable ADR method, it is crucial to be able to accurately predict Envisat's rotation during the mission's time frame. Envisat is currently in a low Earth orbit at an altitude of 770 km (N2YO Webpage, 2006). At this altitude, the main perturbation that affects Envisat is the gravity gradient. The inertia tensor, in its principal axes of inertia, considered for Envisat is shown in equation (43) (Bastida et al., 2014).

$$
I_{0}=\left[\begin{array}{ccc}
129180.25 & 0 & 0 \\
0 & 124801.21 & 0 \\
0 & 0 & 16979.74
\end{array}\right] \mathrm{kg} \cdot \mathrm{m}^{2}
$$

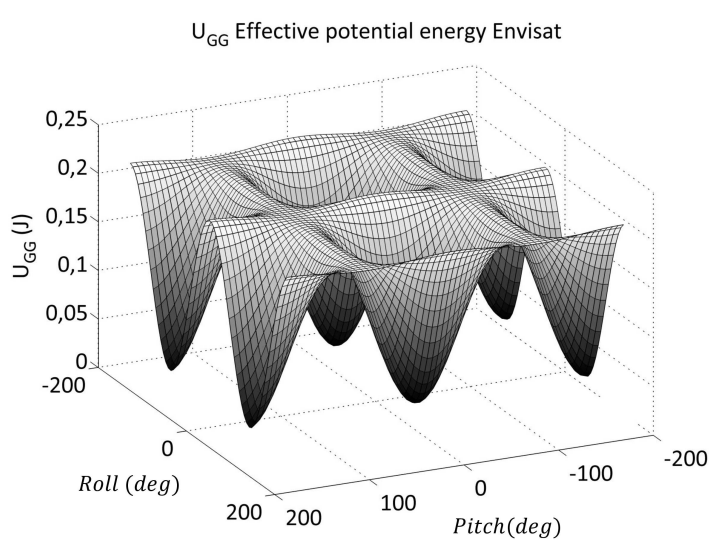

Figure 4: Envisat $U_{G G}$ function

For this spacecraft, an upper limit of the threshold of the non-inertial potential energy $U_{N I}$ is computed. The maximum absolute and minimum absolute of the $U_{N I}$ function are known (see section 3.3). Therefore, an upper limit of the threshold of the non-inertial potential energy $U_{N I}$ can be computed as defined in (44).

$$
\left.\Delta U_{N I}=U_{N I}\left(\phi_{U_{N I \max }}, \theta_{U_{N I \max }}\right)\right)-U_{N I}\left(\phi_{U_{N I m i n}}, \theta_{U_{N I m i n}}\right)
$$

For Envisat's altitude and inertia matrix, the $U_{G G}$ potential energy is portrayed in figure 4 . The valleys of the $U_{G G}$ function correspond to the expected equilibrium positions of the minimum axis of inertia pointing towards nadir and the maximum axis of inertia perpendicular to the orbital plane.

The results of two different simulations are described below in order to show the possible bounded and unbounded motions. It should be highlighted that the initial conditions chosen for the spacecraft (attitude and angular velocity) are not based on real data and were only selected to show the two possible behaviours. A third example is included using as initial conditions, real data derived from the last radar observations carried out in 2013.

In the first simulation, Envisat's initial attitude is assumed to be close to one of the equilibrium points and its kinetic energy is low enough to be trapped within the area of attraction of that equilibrium point. The initial conditions for the simulation were:

- Rotational velocity in the inertial reference frame $\vec{\omega}_{0}=[0.05,0.05,0.05] \mathrm{deg} / \mathrm{sec}$

- Initial attitude (Yaw,Pitch,Roll $)=[0.3,1,88]$ degrees

The initial state gives an initial kinetic energy, $K_{N I}$, equal to $K_{N I}=0.2541 \mathrm{~J}$ which is below the threshold (44). This is a necessary condition, but not sufficient, for the system to have a bounded motion. Two different representations were used to visualise the evolution of Envisat's 

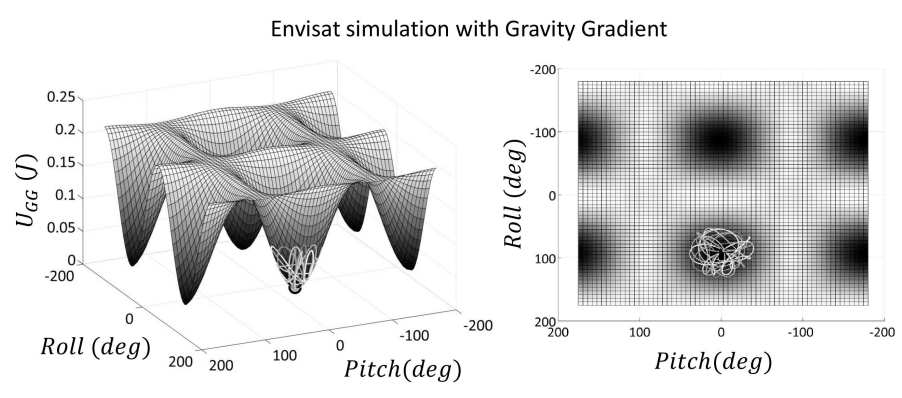

Figure 5: Case 1 Simulation of Envisat with gravity gradient: $U_{G G}(\theta, \phi)$

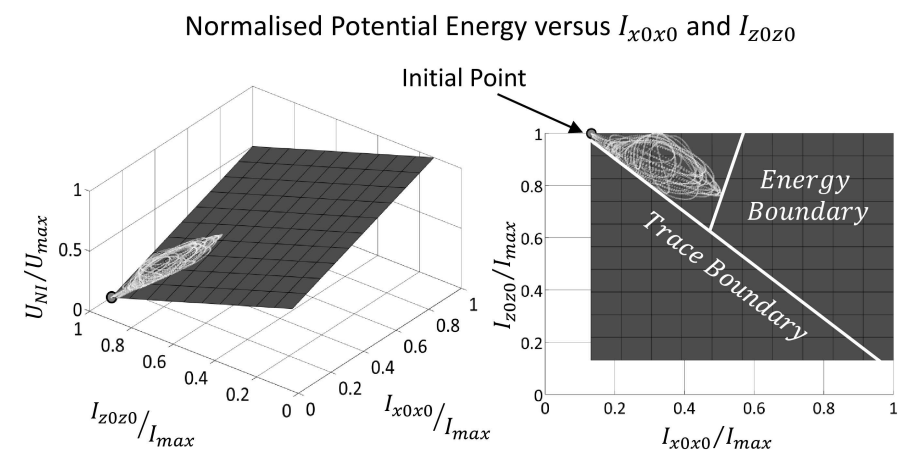

Figure 6: Case 1 Simulation of Envisat with gravity gradient: $U_{N I}\left(I_{x 0 x 0}, I_{z 0 z 0}\right)$

rotational state and check if the motion of the system is bounded. Figure 5 shows how the $U_{G G}(\theta, \phi)$ function of the system evolves. It is observed that the attitude of the spacecraft is confined and the system oscillates around the equilibrium point. In addition, figure 6 plots the function $U_{N I}\left(I_{x 0 x 0}, I_{z 0 z 0}\right)$. In this graphic, the system also remains in the vicinity of the equilibrium point which corresponds to the minimum value of $I_{x 0 x 0}$ and the maximum value of $I_{z 0 z 0}$. In this case, one of the trace boundaries and the energy boundaries are active.

For the second simulation, the kinetic energy is increased and the system no longer stays in the vicinity of an equilibrium point. The initial conditions considered for the second simulation are:

- Rotational velocity in the inertial reference frame $\vec{\omega}_{0}=[1,1,1] \mathrm{deg} / \mathrm{sec}$

- Initial attitude $($ Yaw,Pitch,Roll $)=[0.3,100,88]$ degrees

The initial state gives an initial kinetic energy $K_{N I}$ equal to $K_{N I}=45.58 \mathrm{~J}$ which is far above the threshold (44) and it will give rise to an unbounded motion. Figure 7 shows the evolution of the system in the $U_{G G}$ map. In this case, the system is no longer confined and moves freely within the map. Figure 8 shows the evolution of the system in the plane $U_{N I}\left(I_{x 0 x 0}, I_{z 0 z 0}\right)$ which is constrained by the inferior trace boundary and the rest of the boundaries are
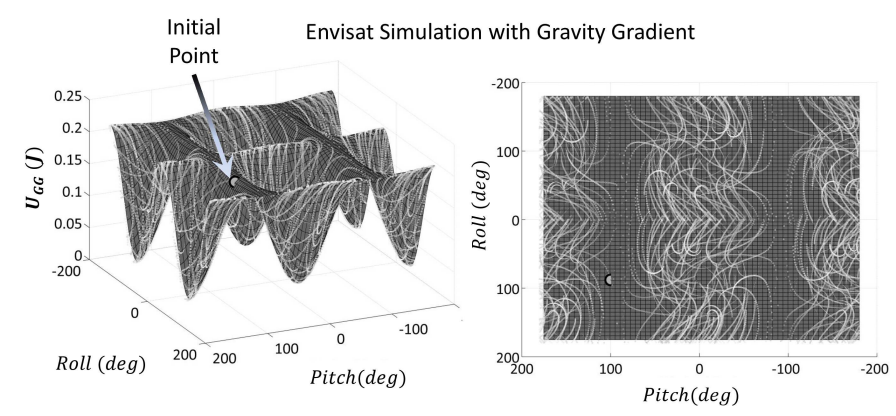

Figure 7: Case 2 Simulation of Envisat with gravity gradient: $U_{G G}(\theta, \phi)$
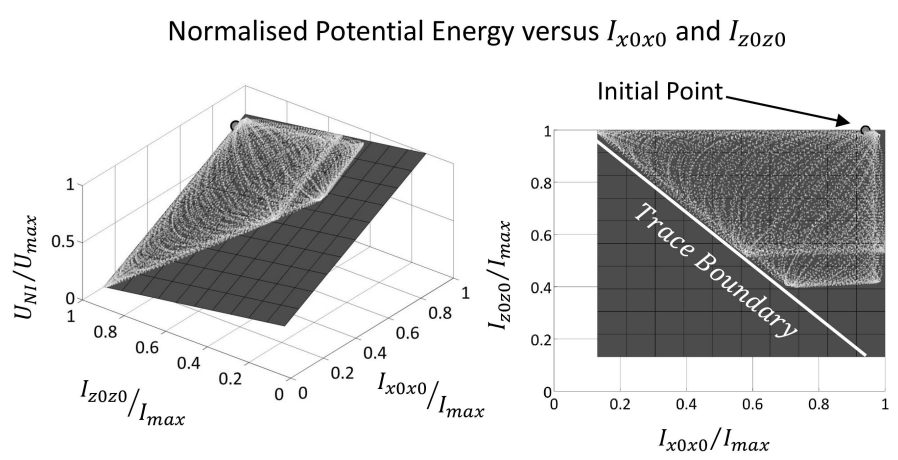

Figure 8: Case 2 Simulation of Envisat with gravity gradient: $U_{N I}\left(I_{x 0 x 0}, I_{z 0 z 0}\right)$

not active. The system does not fill all the allowed domain due to the initial conditions.

The most up-to-date published measurements on the rotational speed of Envisat are the measurements dated September $25^{\text {th }} 2013$ (Kucharski et al., 2014), which was considered to initialise the following simulation. The measured spin rate of Envisat on the 25 ${ }^{\text {th }}$ of September 2013 was $2.67 \mathrm{deg} / \mathrm{sec}$. The inertial spin axis direction was perpendicular to the along track direction $Y_{0}$ and formed an angle of 62 degrees with the nadir direction $X_{0}$. The spin axis was in the positive quadrant of the plane defined by $X_{0}, Z_{0}$. Regarding the initial attitude, it was assumed the spin axis to be parallel to the maximum axis of inertia of the spacecraft and the minimum axis of inertia pointing in the along track direction.

- Initial rotational velocity in the inertial reference frame $\vec{\omega}_{0}=[1.2535,0,2.3575] \mathrm{deg} / \mathrm{sec}$

- Initial attitude (Yaw,Pitch,Roll $)=[90,62,0]$ degrees

The initial state gives an initial kinetic energy, $K_{N I}$, equal to $K_{N I}=140.06 \mathrm{~J}$ which is far above the threshold (44) and it will give rise to an unbounded motion. This will occur regardless of the initial attitude of the spacecraft. Therefore, just under the effect of the gravity gradient, the system will never stabilise within the equilibrium state given by the $U_{N I}$ function (minimum axis of inertia pointing towards the Earth and maximum axis of inertia 


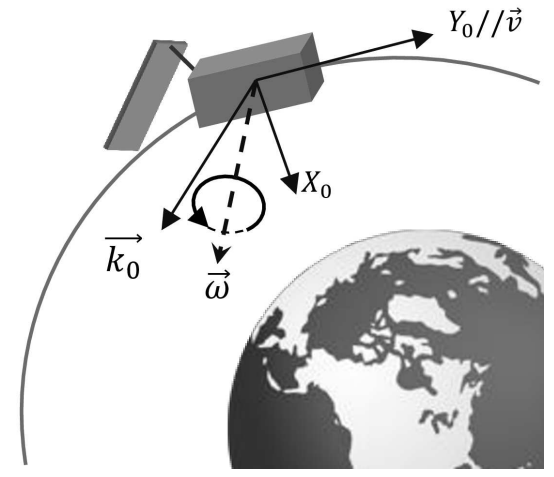

Figure 9: Envisat attitude observed in 2013 (Kucharski et al., 2014).
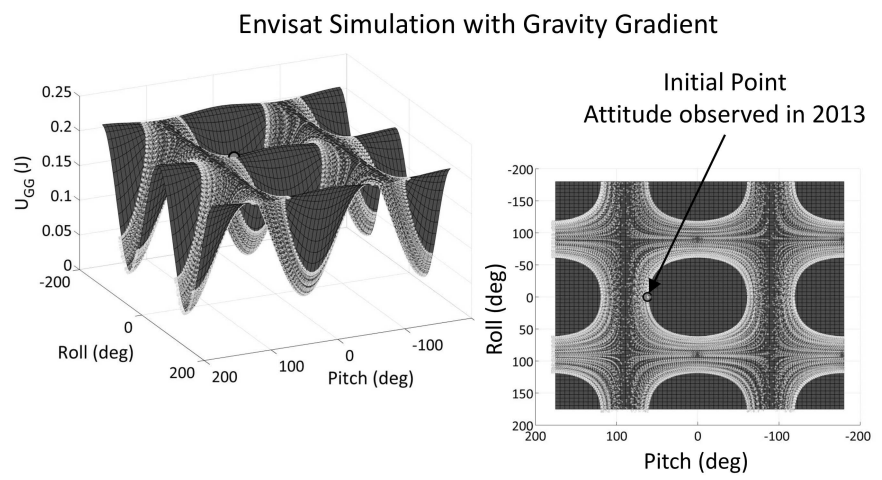

Figure 10: $U_{G G}(\theta, \phi)$ evolution of Envisat with gravity gradient: Initial data from 2013 observations

perpendicular to the orbit). Instead, the gravity gradient can abruptly alter the attitude and spin axis of the spacecraft, as long as the total energy of the system is conserved. This behaviour is clearly verified by the evolution of the effective energy potential $U_{G G}(\theta, \phi)$ in figure 10 .

Figure 11 shows the evolution of the total energy potential $U_{N I}\left(I_{x 0 x 0}, I_{z 0 z 0}\right)$. The system is bounded by the inferior trace boundary while the energy boundary is outside the allowed domain and, thus, it is not active. The system only fills part of the allowed domain due to the initial conditions.

\subsubsection{Case study: spin stabilised spacecraft}

Despite the fact that the only real case studied in the article is Envisat, it was deemed appropriate to include a second example of theoretical nature, to display some peculiarities of the stability problem. In this case, it will be observed that the equilibrium positions are not limited to isolated points but rather to linear connected domains.

The behaviour of the system with regard to the gravity gradient changes with the inertia of the spacecraft. Spin stabilised spacecraft usually have one axis of inertia greater than the other two. In this example the following inertia

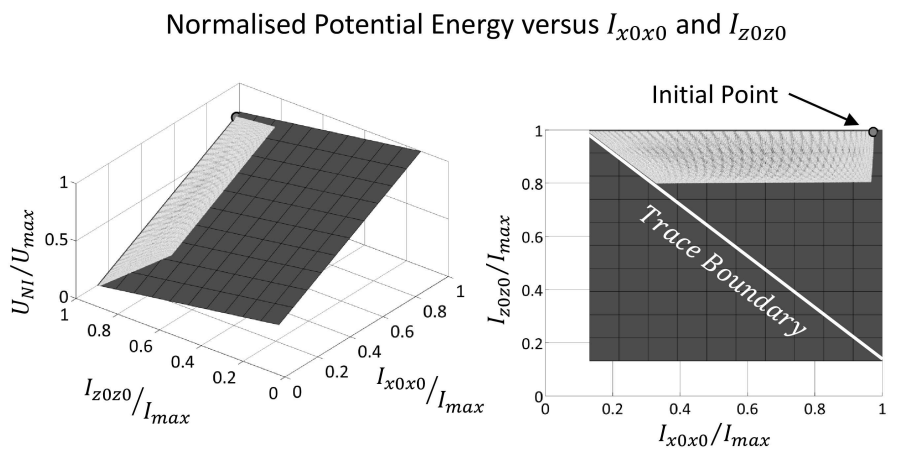

Figure 11: $U_{N I}\left(I_{x 0 x 0}, I_{z 0 z 0}\right)$ evolution of Envisat with gravity gradient: Initial data from 2013 observations

tensor was considered:

$$
I_{0}=\left[\begin{array}{ccc}
1000 & 0 & 0 \\
0 & 1000 & 0 \\
0 & 0 & 10000
\end{array}\right] \mathrm{kg} \cdot \mathrm{m}^{2} .
$$

For this spacecraft, an upper limit of the $U_{N I}$ function can be computed subtracting the absolute maximum and minimum values of the function:

$$
\left.\Delta U_{N I}=U_{N I}\left(\phi_{U_{N I \text { max }}}, \theta_{U_{N I \text { max }}}\right)\right)-U_{N I}\left(\phi_{U_{N I \text { min }}}, \theta_{U_{N I \text { min }}}\right)
$$

Figure 12 shows the shape of the $U_{G G}$ function for this type of satellite. The orbital height considered to obtain this function was $760 \mathrm{~km}$. Instead of local minima, the function has linear varieties where $U_{G G}$ acquires its minimum value. The system will have an unpredictable motion moving freely through the map with minimum potential energy. The initial attitude and rotational velocity considered for the spacecraft are listed below.

- Rotational velocity in the inertial reference frame $\vec{\omega}_{0}=[0.5,0.5,0.5] \mathrm{deg} / \mathrm{sec}$

- Initial attitude $($ Yaw,Pitch,Roll $)=[0.1,0.1,0.1]$ degrees

The initial state gives an initial kinetic energy, $K_{N I}$, equal to $K_{N I}=0.029 \mathrm{~J}$ which is below the threshold (46). The simulations reveal that the system does not possess enough kinetic energy to reach the peaks of the energy potential function. However, due to the absence of local minima points, the system can move with a certain degree of freedom. This behaviour can be clearly observed in figure 13. Figure 14 indicates that the inertia $I_{x 0 x 0}$ remains close to its minimum value and $I_{z 0 z 0}$ remains close to its maximum value which is in line with figure 13, where the potential energy remains close to its minimum value.

\section{Eddy currents}

The metallic structure along with the subsystem components is assumed to be a rigid body and therefore moves 


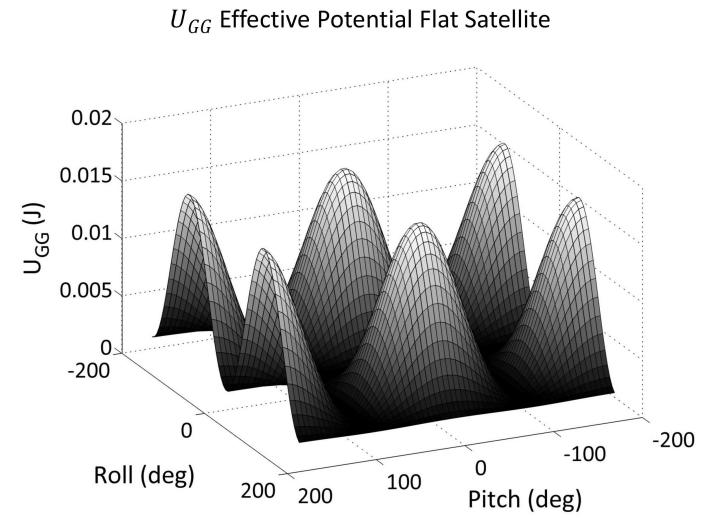

Figure 12: Flat Satellite $U_{G G}$ function

Flat Satellite Simulation with Gravity Gradient
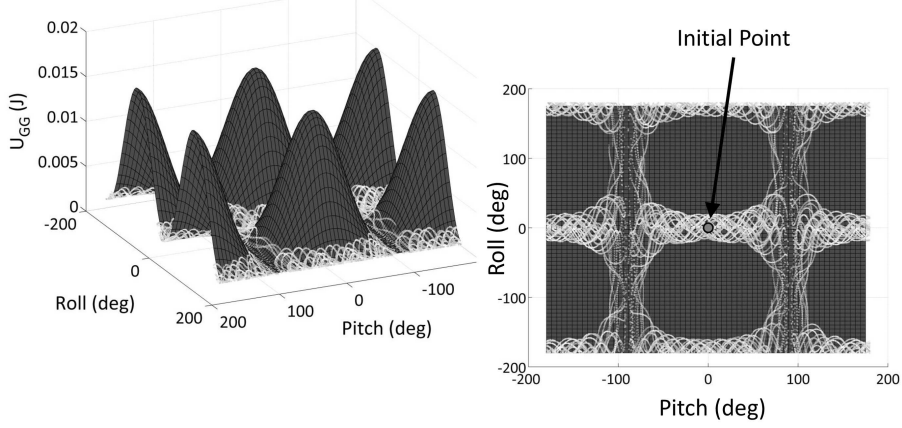

Figure 13: Flat Satellite simulation with gravity gradient: $U_{G G}(\theta, \phi)$ evolution
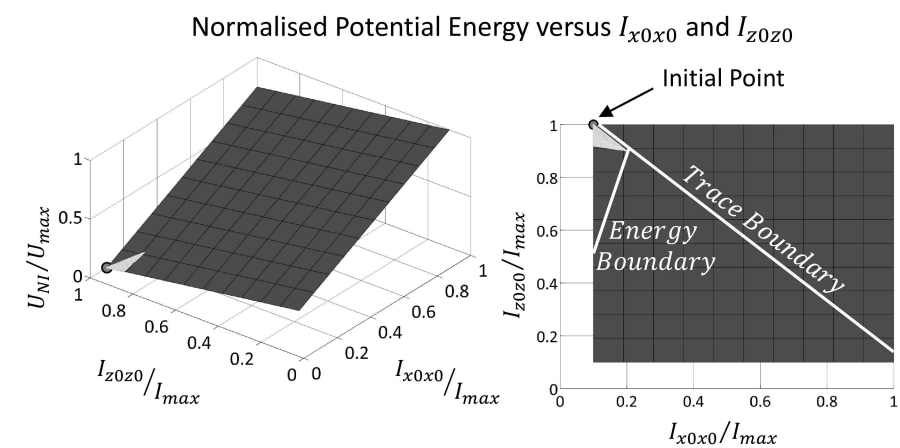

Figure 14: Flat Satellite simulation with gravity gradient: $U_{N I}\left(I_{x 0 x 0}, I_{z 0 z 0}\right)$ evolution as a single entity. Due to the existence of an external magnetic field, the rotation of the object induces electric currents following Lenz's Law. These currents are responsible for a dissipative effect caused by Joule's Law which translates into a torque that opposes to the general rotational movement of the object.

In this work, a model of the metallic structure is defined within the simulation process. Equation (55) is used to compute the magnetic tensor $M$, which is constant throughout the integration process in the body reference frame. Using this tensor, equation (50) provides the torque in each time step of the simulation. Finally, this torque is introduced as a new term in the Euler equations and the dissipative effects explained throughout the article are thus observed. In section 4.1, the mathematical models that allow the induced torque to be evaluated are developed. Later on, their main effects on space debris objects are explained and finally, a case study on the Envisat spacecraft is presented.

\subsection{General equations}

This perturbation is caused by the Earth's magnetic field and therefore, it is only noticeable in LEO. When a conductive rotating body is under the effect of a magnetic field, eddy current loops are induced in the object (Landau, 1984). This generates torques which decrease the rotational speed of the target and that may also cause a precession of the object's axis of rotation (Ormsby, 1967).

In order to study this phenomenon, the space debris object is assumed to be a rigid body and the conductive materials are assumed to have a relative permeability close to 1. Typical metallic structures of spacecraft and upper stages are made of this type of material (i.e., aluminum, titanium, copper). Under these assumptions, the general equations that describe this phenomenon are (Praly et al., 2012; Landau, 1984) shown in (47), (48) and (49) as,

$$
\begin{gathered}
\nabla \cdot \vec{j}=0 \quad \forall P \in V, \\
\nabla \times \vec{j}=\sigma(\vec{\omega} \times \vec{B}) \quad \forall P \in V, \\
\vec{j} \cdot \vec{n}_{v}=0 \quad \forall P \in \partial V,
\end{gathered}
$$

where $\vec{j}$ is the electric current density vector that is induced in the volume $V, \vec{\omega}$ is the rotational velocity vector of the object, $\vec{B}$ is the magnetic field, $\sigma$ is the conductivity of the body and $\vec{n}_{v}$ is a vector normal to the contour surface $\partial V$.

Unlike the gravity gradient, the eddy currents phenomenon is a dissipative effect. The derivative of the kinetic energy $\dot{K}$ is always negative or equal to zero. This is explained by Joule's effect as the kinetic energy of the system is transformed into heat and the rotational velocity of the object is damped (Landau, 1984).

The general formula of the torque induced by the eddy currents is defined in (50),

$$
\vec{T}=(M(\vec{\omega} \times \vec{B})) \times \vec{B},
$$


where $M$ is the magnetic Tensor defined in Ortiz \& Walker (2014). The formula for the eddy currents torque (50) generalises the analytical solutions given by previous authors ((Ormsby, 1967), (Smith, 1962)).

The general solution of the magnetic tensor can be derived from equations $(47,48,49)$ together with Joule's Law which can be expressed as a function of the magnetic tensor as shown in (51).

$$
\dot{K}=-\Omega^{t} M \Omega \leq 0=-\int \frac{j^{2}}{\sigma},
$$

where $\vec{\Omega}=\vec{\omega} \times \vec{B}$.

Equations $(47,48,49)$ can be solved with a generic F.E.M. which will give the solution for $\vec{j}$ at the gaussian points of the elements of the grid. Solving these equations for the three cases $\Omega^{(1)}=\left[\begin{array}{l}1 \\ 0 \\ 0\end{array}\right], \Omega^{(2)}=\left[\begin{array}{l}0 \\ 1 \\ 0\end{array}\right], \Omega^{(3)}=\left[\begin{array}{l}0 \\ 0 \\ 1\end{array}\right]$, a set of solutions for the vector $\vec{j}^{(k)}(\vec{r})$ is obtained. Taking into account that the solution of $\vec{j}$ is linear in $\vec{\Omega}=\vec{\omega} \times \vec{B}$ (see equations $(47,48,49))$, the solution for a given $\Omega=\left[\begin{array}{l}\Omega_{1} \\ \Omega_{2} \\ \Omega_{3}\end{array}\right]$ acquires the form:

$$
\vec{j}=\sum_{k=1}^{3} \Omega_{k} \cdot \vec{j}^{(k)}(\vec{r}) .
$$

Finally, entering in equation (51), the following formula for the magnetic tensor is reached:

$$
M_{k l}=\sum_{(e l)} \int_{V^{(e l)}} \frac{\vec{j}^{(k)} \cdot \vec{j}^{(l)}}{\sigma} d V
$$

In addition, an explicit formula for the magnetic tensor $M$ can be obtained using the most simple finite element, which is a straight two-nodes bar. The key to reach this formula is to express the general solution for equations $(47,48,49)$ as:

$$
\begin{array}{r}
\vec{j}=\vec{j}_{\text {part }}-\sigma \nabla \psi, \\
\vec{j}_{\text {part }}=\frac{\sigma}{2}(\omega \times \vec{B}) \times \vec{r},
\end{array}
$$

where $\psi$ is an unknown scalar function and $\vec{r}$ is a state position vector of the elements of the F.E.M. The space debris object is divided in $m$ bars and $n$ nodes and the following matrices must be computed:

- $D$ is a diagonal matrix of dimensions $m \times m$ that contains the constitutive constants of the bars that form the grid. The constitutive constant of each bar is $D(k, k)=\frac{\sigma_{k} A_{k}}{L_{k}}(k=1, . ., m)$, where $\sigma_{k}$ is the conductivity, $A_{k}$ is the cross section and $L_{k}$ is the length of the bar.
- $S$ is a matrix of dimensions $m \times 3$ that contains the cross products $\vec{r}_{k} \times \vec{L}_{k}(k=1, . ., m)$, where $\vec{r}_{k}$ is the position of the COG of each bar and $\vec{L}_{k}=L_{k} \vec{l}_{k}$ is a vector in the direction of the bar and norm equal to its length.

- $I$ is the identity matrix of dimensions $m \times m$.

- $F=H^{t}\left(H D H^{t}\right)^{-1} H$ where $H$ is a matrix of dimensions $m \times n$ that indicates the connectivity of the nodes in the grid. The term $H(k, l)$ of the matrix will be 1 if the intensity of the bar $J_{k}$ is entering the node $l,-1$ if the intensity is leaving the node and zero if the intensity $J_{k}$ does not flow through node $l$.

Using the previous parameters of the grid, the general solution for the magnetic tensor is:

$$
M=\frac{1}{4} S^{t} D(I-F D) D^{-1}(I-D F) D S .
$$

\subsection{Characteristic time of decay}

Equation (50) shows that if the rotational velocity vector $\vec{\omega}$ is parallel to the magnetic field $\vec{B}$, there is no induced torque. Therefore, only the perpendicular component of the magnetic field is damped. If there is an additional component parallel to the magnetic field, the object will precess its axis of rotation until the rotational velocity vector is parallel to the magnetic field. By introducing the eddy currents torque into the Euler equations, the characteristic time of decay can be inferred,

$$
\begin{gathered}
I \dot{\vec{\omega}}+(\vec{\omega} \times I \vec{\omega})=(M(\vec{\omega} \times \vec{B})) \times \vec{B}, \\
\tau_{i} \approx \frac{I_{i}}{M_{j} B_{\perp}^{2}}, \quad 1 \leq i \neq j \leq 3 .
\end{gathered}
$$

Each characteristic time of decay $\tau_{i}$ corresponds to three independent cases of the body rotating about a theoretical uniaxial axis equal to each principal axis of inertia. This value will give an estimate of the expected time of decay along each principal axis. $I_{i}$ and $M_{j}$ are the corresponding components of the inertia tensor and magnetic tensor of the object. $B_{\perp}$ is the perpendicular component of the magnetic field with respect to each principal axis. The ratio $I_{i} / M_{j}$ accounts for the amount of conductive material that is present on the spacecraft.

\subsection{Stability analysis}

Once the dissipative effect (eddy currents) has been added on top of a conservative effect (gravity gradient), the previous region defined as 'region of marginal stability' might be referred to now as a 'basin of attraction'. The eddy currents phenomenon will make the system lose kinetic energy $K_{N I}$ with time and the system may eventually converge towards a local minimum of the potential energy function $U_{N I}$. In this case, the system is now asymptotically stable. If the potential energy $U_{N I}$ has different local 


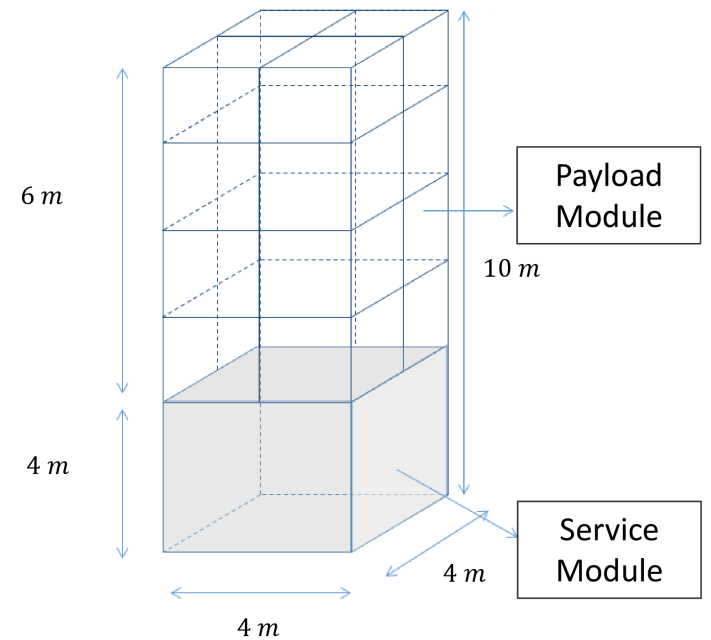

Figure 15: Envisat metallic structure model

minima, the system may converge to one equilibrium point or another depending on the initial conditions.

Nonetheless, the example considered in (3.4.2) shows the need for flexibility in this type of lexicon because the real debris may disconcert in for their diversity of qualitative behaviours.

\subsection{Case study: Envisat}

The magnetic tensor of Envisat was obtained with the Finite Element Method model presented in figure 15. This model accounts for the effective metallic material which will generate eddy currents (e.g. shielded components will not be affected by the magnetic field and the contribution from unconnected plates is disregarded). Most of the metallic structural material is concentrated on the service module while the structural materials of the payload module are mainly composed of composite materials (ESA, 1998). The service module structure was modeled as an aluminum cubical shell of $4 \times 4 \times 4 \mathrm{~m}^{3}$ and an aluminum thickness of $0.3 \mathrm{~mm}$. For the payload module, it was considered that the composite structure has an aluminum frame. The frame was modeled with circular bars of $40 \mathrm{~mm}$ in diameter and $0.3 \mathrm{~mm}$ of thickness. All these materials are assumed to be electrically connected and assumed to have a conductivity of $3.5 \mathrm{e} 7 \mathrm{~S} / \mathrm{m}$. Therefore the conservative estimate of the total amount of effective conductive material is $2.5 \%$ of the total mass of the spacecraft $7827.9 \mathrm{~kg}$ (Bastida et al., 2014). Under these conditions, the magnetic tensor obtained for Envisat is shown in (58).

$$
M=\left[\begin{array}{ccc}
1.0590 & 0 & 0 \\
0 & 1.0590 & 0 \\
0 & 0 & 0.9315
\end{array}\right] \cdot 10^{6} \mathrm{~S} \cdot \mathrm{m}^{4}
$$

A simulation was carried out including the Earth's gravity gradient and magnetic field. The Earth's magnetic field
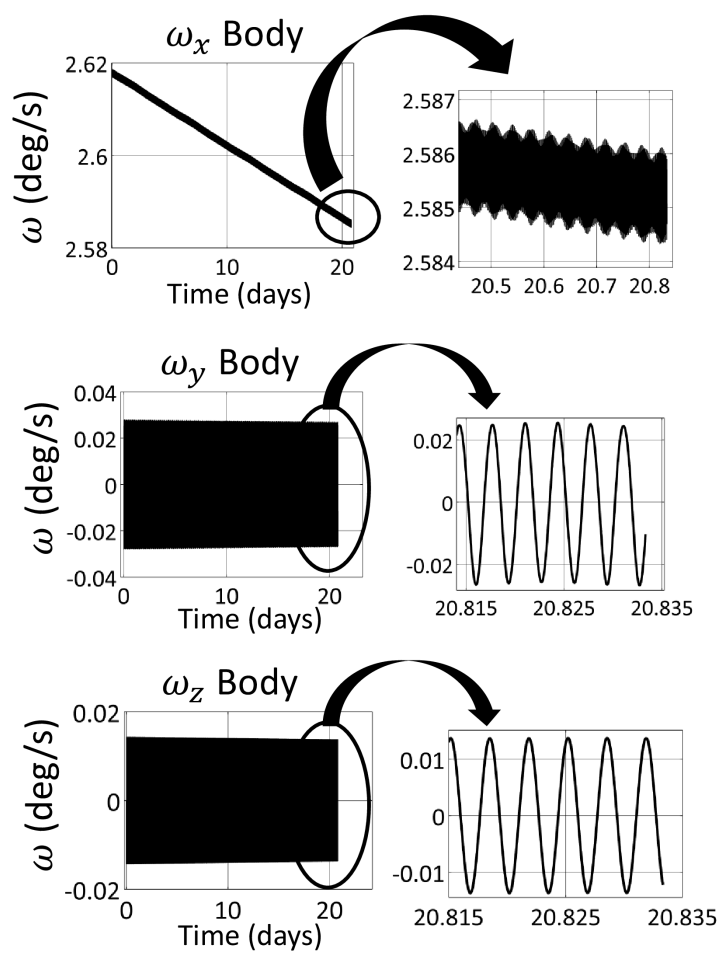

Figure 16: Envisat rotational velocity between inertial and body reference frames, expressed in the body frame.

was modeled based on the International Geomagnetic Reference Field (IGRF-11). Envisat is placed at a circular orbit at an altitude and inclination of $770 \mathrm{~km}$ and 98.4 degrees respectively (N2YO Webpage, 2006) with the following initial conditions (Kucharski et al., 2014).

- Initial rotational velocity in the inertial reference frame $\vec{\omega}_{0}=[1.2535,0,2.3575] \mathrm{deg} / \mathrm{sec}$

- Initial attitude (Yaw,Pitch,Roll $)=[90,62,0]$ degrees

- Initial date: 25-September-2013

- Propagation Time: 20 days

Figure 16 shows the three components of the rotational velocity vector between the body frame and the inertial reference frame expressed in the body frame of the spacecraft. The major contribution of the rotational speed goes along the $X_{b}$ axis (maximum axis of inertia of the spacecraft) and it can be observed how this component decreases with time. The other two components start oscillating due to the change in direction of the Earth's magnetic field along the orbit and the Earth's gravity gradient. These two components also decrease with time but at a lower rate.

The kinetic energy decreases with time and the system will converge for $t \rightarrow \infty$ to a local minima of the potential energy function. This behaviour is observed in figure 17 where the function $U_{G G}(\theta, \phi)$ is depicted. A color code was used to show the time evolution of the system, starting 

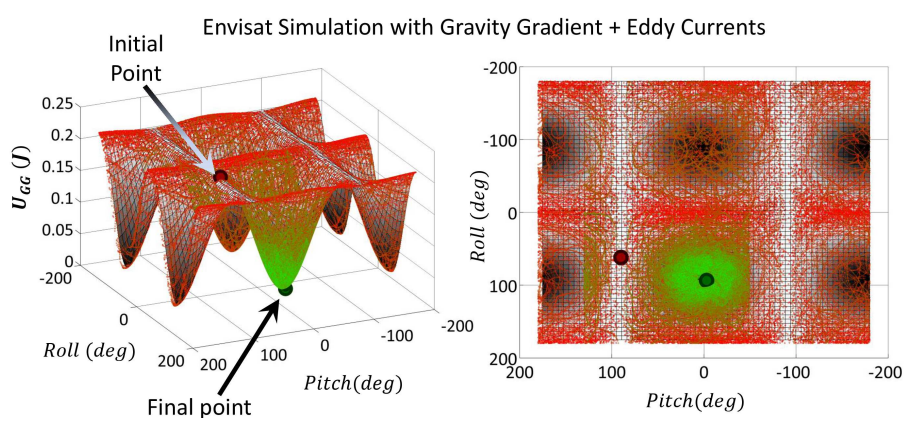

Figure 17: Envisat simulation with gravity gradient and eddy currents: $U_{G G}(\theta, \phi)$ evolution.

in red and finalising the simulation in green. The initial conditions of the attitude and angular velocity determine which local minima the system converges to. In the case of Envisat, for $t \rightarrow \infty$, the rotational velocity decreases to zero and the attitude of the spacecraft will point its minimum axis of inertia towards the Earth and its maximum axis of inertia towards the axis perpendicular to the orbital plane.

The simulation also provided the mean value of the Earth's magnetic field along the principal axis of inertia of the spacecraft as shown in (59).

$$
\vec{B}_{\text {mean }}=[9.1,17.9,17.9] \mu T .
$$

The mean value obtained for the Earth's magnetic field was used to obtain three representative values of the characteristic time of decay along the principal axes of inertia of the body. Each characteristic time of decay $\tau_{i}$ corresponds to three theoretical cases in which the body is assumed to have a uniaxial rotation along one of the principal axis of inertia. For each principal axis, the corresponding perpendicular component of $B_{\text {mean } \perp}$ was considered and the following values were obtained:

$$
\begin{gathered}
\tau_{1}=2277.6 \text { days. } \\
\tau_{2}=3475.6 \text { days. } \\
\tau_{3}=541.9 \text { days. }
\end{gathered}
$$

For each of the three cases, the spin period at a certain time $t$ is:

$$
\omega=\frac{2 \pi}{T} \rightarrow T(t)=T_{0} \exp \left(\frac{t}{\tau}\right)
$$

Therefore, the spin period change per unit time around a certain date is:

$$
\dot{T}=\frac{T(t)}{\tau} .
$$

Kucharski at al.,(Kucharski et al., 2014), measured a spin period increase of $36.7 \mathrm{~ms} /$ day between day 100 and day 270 in the year 2013. The spin period increase is evaluated at the central point of the measurements carried out in 2013, which corresponds to a spin period of $T_{0}=$
131.678 seconds. Using this value and the three different $\tau_{i}$, the spin period rate $\dot{T}_{i}$ is computed:

$$
\begin{gathered}
\dot{T}_{1}=59.6 \mathrm{~ms} / \text { days. } \\
\dot{T}_{2}=38.9 \mathrm{~ms} / \text { days. } \\
\dot{T}_{3}=251.1 \mathrm{~ms} / \text { days. }
\end{gathered}
$$

These results may be viewed as representative values of the period growth, depending on the variable attitude of the satellite. The values are consistent with the measurements carried out in 2013 and the eddy currents induced by the Earth's magnetic field are a possible explanation for the current rotational speed decrease of Envisat. In order to obtain more accurate simulations, a detailed description of the structural materials and components as well as their conductive connectivity is necessary. This would allow a better approximation of the magnetic tensor to be obtained for the Envisat spacecraft.

\section{Conclusion}

The understanding and accurate prediction of the probable rotational states of space debris objects is crucial in the design of active debris removal missions. Current ADR methods that require physical contact with the target have important physical limitations depending on the rotational speed and spin motion of the object. Therefore, an accurate prediction of the rotational state at the time of capture is necessary in order to choose the most suitable ADR method and also to clarify if a de-tumbling phase prior to the capturing phase is necessary. In this article the effect of the Earth's gravity gradient and the eddy currents induced by the Earth's magnetic field are studied for the analysis of the rotational dynamics of space debris objects. These two perturbations are noticeable in Low Earth Orbits for objects which have a non-isotropic inertia tensor (in the case of the gravity gradient) and which have conductive materials (in the case of the eddy currents). A graphical interpretation of the gravity gradient is presented and two possible patterns are encountered. The first one is a 'bounded motion' in which the variation of the object's attitude is confined to a certain region of marginal stability. In this pattern, the attitude oscillates around a certain equilibrium state. The second possible pattern is an 'unbounded motion' in which the attitude of the body is not confined and the system will jump between different equilibrium states. This will occur when the kinetic energy is above a certain threshold given by the equation of the conservation of total energy. These two patterns will only occur if the inertia tensor has the right properties. Other more complicated patterns exist when the equilibrium states are not points but linear varieties. In addition, an analysis was carried out for the Envisat spacecraft that became uncontrollable in April 2012. The rotational speeds measured in 2013 indicate that Envisat finds itself in an 'unbounded motion' and it will not be stabilised by the gravity gradient unless additional dissipative 
effects act on the object. The second part of the article focused on the analysis of the natural eddy currents induced by the Earth's magnetic field. This is a dissipative effect that appears on conductive bodies rotating in the presence of a magnetic field. It will exponentially decrease the perpendicular component of the rotational velocity with respect to the Earth's magnetic field vector. It may cause a precession of the axis of rotation if there is a component of the rotational velocity vector parallel to the magnetic field. Combining both the Earth's gravity gradient and the Earth's magnetic field, the rotational speed of space debris objects has been shown to decrease. This phenomenon is a possible explanation for the rotational speed decrease rate which was observed between April 2013 and September 2013 for the Envisat spacecraft.

\section{A. Inertia matrix expressed in the orbital refer- ence frame}

The relationship between the inertia matrix expressed in the orbital reference frame and the orbital reference frame is:

$$
I_{0}=R_{b \rightarrow 0} I_{b} R_{b \rightarrow 0}^{T},
$$

where $I_{0}$ is the inertia matrix expressed in the orbital frame, $I_{b}$ is the inertia matrix expressed in the body frame and $R_{b \rightarrow 0}$ is the rotation matrix to go from the body frame to the orbital reference frame (1).

The inertia matrix in the orbital frame is:

$$
I_{0}=\left[\begin{array}{ccc}
I_{x 0 x 0} & I_{x 0 y 0} & I_{x 0 z 0} \\
I_{x 0 y 0} & I_{y 0 y 0} & I_{y 0 z 0} \\
I_{x 0 z 0} & I_{y 0 z 0} & I_{z 0 z 0}
\end{array}\right]
$$

Using equation (64), the relationship between the inertias is obtained:

$$
I_{x 0 x 0}=I_{x x} \cos ^{2} \theta \cos ^{2} \phi+I_{y y} \sin ^{2} \theta+I_{z z} \cos ^{2} \theta \sin ^{2} \phi,
$$

$$
\begin{array}{r}
I_{y 0 y 0}=I_{x x}(\cos \phi \sin \theta \cos \psi+\sin \phi \sin \psi)^{2}+ \\
I_{y y}(\cos \psi \cos \theta)^{2}+ \\
I_{z z}(\sin \phi \sin \theta \cos \psi-\cos \phi \sin \psi)^{2}, \\
I_{z 0 z 0}=I_{x x}(\cos \phi \sin \theta \sin \psi-\sin \phi \cos \psi)^{2}+ \\
I_{y y}(\sin \psi \cos \theta)^{2}+ \\
I_{z z}(\sin \phi \sin \theta \sin \psi+\cos \phi \cos \psi)^{2},
\end{array}
$$

$I_{x 0 y 0}=I_{x x}(\cos \theta \cos \phi)(\cos \phi \sin \theta \cos \psi+\sin \phi \sin \psi)-$ $I_{y y} \sin \phi \cos \psi \cos \theta+$ $I_{z z} \sin \phi \cos \theta(\sin \phi \sin \theta \cos \psi-\cos \phi \sin \psi)$,

$$
\begin{array}{r}
I_{x 0 z 0}=I_{x x} \cos \theta \cos \phi(\cos \phi \sin \theta \sin \psi-\sin \phi \cos \psi)- \\
I_{y y} \sin \theta \sin \psi \cos \theta+ \\
I_{z z} \sin \phi \cos \theta(\sin \phi \sin \theta \sin \psi+\cos \phi \cos \psi),
\end{array}
$$

$$
\begin{array}{r}
I_{y 0 z 0}=I_{x x}(\cos \phi \sin \theta \cos \psi+\sin \phi \sin \psi) \\
(\cos \phi \sin \theta \sin \psi-\sin \phi \cos \psi) \\
+I_{y y} \cos \psi \cos ^{2} \theta \sin \psi \\
+I_{z z}(\sin \phi \sin \theta \cos \psi-\cos \phi \sin \psi) \\
(\sin \phi \sin \theta \sin \psi+\cos \phi \cos \psi),
\end{array}
$$

\section{B. Non-inertial term of the energy equation due to the gravity gradient effect}

In this section, it is demonstrated that the next relationship holds:

$$
-\frac{\Delta E}{d t}=\vec{T} \cdot \overrightarrow{\omega_{n}}, \Delta E=-\left(I \vec{\omega}_{b i}\right) \cdot \vec{\omega}_{n} .
$$

The derivative of $\Delta E$ is:

$$
\begin{gathered}
\frac{\Delta E}{d t}=n I \dot{\vec{\omega}}_{b i} \cdot \vec{k}_{0}+n I \vec{\omega}_{b i} \cdot \dot{\vec{k}}_{0}= \\
n I \dot{\vec{\omega}}_{b i} \cdot \vec{k}_{0}-n I \vec{\omega}_{b i} \cdot\left(\vec{\omega}_{b i} \times \vec{k}_{0}\right)
\end{gathered}
$$

where the following expression of the derivative of $\vec{k}_{0}$ in body axes has been used:

$$
\dot{\vec{k}}_{0}=-\vec{\omega}_{b 0} \times \vec{k}_{0}=-\vec{\omega}_{b i} \times \vec{k}_{0}
$$

as $-\vec{\omega}_{0 i} \times \vec{k}_{0}=\overrightarrow{0}$.

Now, using Euler equations:

$$
I \dot{\vec{\omega}}_{b i}+\vec{\omega}_{b i} \times I \vec{\omega}_{b i}=\vec{T} \rightarrow I \dot{\vec{\omega}}_{b i}=\vec{T}-\vec{\omega}_{b i} \times I \vec{\omega}_{b i}
$$

$$
\frac{\Delta E}{d t}=n\left(\vec{T}-\vec{\omega}_{b i} \times I \vec{\omega}_{b i}\right) \cdot \vec{k}_{0}-n I \vec{\omega}_{b i} \cdot\left(\vec{\omega}_{b i} \times \vec{k}_{0}\right)
$$

Taking into account that $I \vec{\omega}_{b i} \cdot\left(\vec{\omega}_{b i} \times \vec{k}_{0}\right)=\left(I \vec{\omega}_{b i} \times \vec{\omega}_{b i}\right)$. $\vec{k}_{0}$, the former expression reduces to:

$$
\frac{\Delta E}{d t}=\vec{T} \cdot n \vec{k}_{0}=-\vec{T} \cdot \vec{\omega}_{n}
$$

\section{Acknowledgements}

The research work that is being developed at Southampton University in the United Kingdom is part of the European Union Framework 7 Program, Marie Curie Initial Training Networks (ITN) project 'Stardust' (Stardust Program, 2013). This network is led by Professor Massimiliano Vasile at the University of Strathclyde in Glasgow, Scotland. 
Bastida Virgili, B., Lemmens, S., \& Krag, H., Investigation on Envisat Attitude Motion, e.Deorbit Workshop, European Space Agency, The Netherlands, May 2014.

Biesbroek, R., Soares, T., Huesing, J., Wormnes, K., \& Innocenti, L., The e.Deorbit CDF Study: A design study for the safe removal of a large space debris, 64rd International Astronautical Congress (IAC) Beijing, 2013.

European Space Agency, Position Paper on Space Debris Mitigation. Implementing Zero Debris Creation Zones, SP-1301, European Space Agency, The Netherlands, October 2005.

European Space Agency, Envisat-1. Mission and System Summary., ESA Brochure, Issue 2, The Netherlands, March 1998.

Gerlach, O. H., Attitude Stabilization and Control of Earth Satellites, Report VTH 122, Technische Hogeschool Delft Vliegtuigbouwkunde, Delft The Netherlands, March 1965.

Kessler, D.J., Johnson, N.L., Liou, L.C., \& Matney, M., The Kessler Syndrome: Implications to Future Space Operations, 33rd Annual AAS Guidance and Control Conference Paper AAS 10-016, 2010.

Kucharski, D., Kirchner, G., Koidl, F., Fan, C., \& Carman, R., et al., Attitude and Spin Period of Space Debris Envisat Measured by Satellite Laser Ranging, Geoscience and Remote Sensing, IEEE Transactions, Vol 52, Issue 12, pages 7651-7657, December 2014.

Hughes, P.C., Spacecraft Attitude Dynamics, Dover Publications, INC., 2004.

Landau, L.D., \& Lifshitz, E.M., Electrodynamics of continuous media, Laundau and Lifshitz Course of Theoretical Physics Volume 8, Pergamon Press, 1984.

Landau, L.D., \& Lifshitz, E.M., Mechanics. Course of Theoretical Physics Volume 1 Third Edition., Reed Educational and Professional Publishing Ltd., 1976.

Langford, R.C., \& Mundo, C.J., Guidance and Control-II, Progress in Astronautics and Aeronautics Volume 13, 1964, Academic Press, United Kingdom.

Lemmens, S., Krag, H., Rosebrock, J., \& Carnelli, I., Radar Mappings for Attitude Analysis of Objects in Orbit, 6th European Conference on Space Debris, Darmstadt, April 2013.

N2YO Webpage, http://www.n2yo.com/, Real Time Satellite Tracking and Prediction, 2006.

Process for Limiting Orbital Debris, NASA-STD-8719.14A, National Aeronautics and Space Administration, Washington DC, December 2011.

Nishida, S.I., \& Kawamoto, S. Strategy for capturing of a tumbling space debris, Acta Astronautica, Volume 68, Issues 1-2, Pages 113120, January-February 2011.

Ojakangas, G. W., Anz-Meador, P., \& Cowardin, H., Probable Rotation States of Rocket Bodies in Low Earth Orbit, 14th annual Maui Optical and Space and Surveillance Technologies Conference; 11-14 Sep. 2012; Maui, HI; United States.

Ormsby, J. F. A., Eddy Current Torques and Motion Decay on Rotating Shells., Project 8051, United States Air Force, year 1967.

Ortiz Gómez, N., \& Walker, S.J., Eddy Currents applied to detumbling of space debris: analysis and validation of approximate proposed methods, Proceedings of the International Astronautical Congress,IAC-14,A6,6,2,x22528, Toronto, 2014.

Papushev, P., Karavaev, Y., \& Mishina, M. Investigations of the evolution of optical characteristics and dynamics of proper rotation of uncontrolled geostationary articial satellites., Advances in Space Research, Volume 43, Issue 9, pages 1416-1422, year 2009.

Praly, N., Hillion, M., Bonnal, C., Laurent-Varin, J., \& Petit, N., Study on the eddy current damping of the spin dynamics of space debris from the Ariane launcher upper stages, Acta Astronautica, Volume 76, July-August 2012, Pages 145-153.

Siahpush, A., \& Sexton, A., A Study for Semi-passive Gravity Gradient Stabilization of Small Satellites, USU Conference on Small Satellites, October 7-9, 1987.

Smith, L., Effects of Magnetically Induced Eddy-Current Torques on Spin Motions of an Earth Satellite, NASA Technical Note TN D-2198, National Aeronautics and Space Administration, Washington D.C., April 1965.

Smith, L., A Theoretical Study of the Torques induced by a Magnetic Field on Rotating Cylinders and Spinning Thin-wall Cones, Cone
Frustums, and General Body of Revolution, Planetary and Space Science, National Aeronautics and Space Administration, Rep. Number R-129.

Stardust Program Webpage, http://www.stardust2013.eu/, Stardust Program, Advanced Research Network on Asteroid and Space Debris Manipulation 2013.

United Nations Office for Outer Space Affairs, Committee on the Peaceful Uses of Outer Space Active Debris Removal-An Essential Mechanism for Ensuring the Safety and Sustainability of Outer Space, A/AC.105/C.1/2012/CRP.16, Vienna, February 2012.

Williams, V. ,\& Meadows, A.J. Eddy Current Torques, air torques, and the spin decay of cylindrical rocket bodies in orbit, Journal of Planetary and Space Science, Vol. 26, pages 721-726., 1978.

Wormnes, K. , Le Letty, R. ,\& Summerer, L. ESA Technologies for Space Debris Remediation, Presentation of the 6th European Conference on Space Debris, European Space Agency, The Netherlands, 2013. 